\title{
Unusual Multiple Brain Abscesses Caused by Aeromonas hydrophila in a Preterm Neonate: Case Report
}

\author{
Elham Essa Bukhari ${ }^{1}$ \\ ${ }^{1}$ Department of Pediatrics, Infectious Disease Unit, King Saud \\ University, Riyadh, Saudi Arabia \\ J Child Sci 2018;8:e55-e57.
}

\begin{abstract}
Address for correspondence Elham Essa Bukhari, MD, Department of Pediatrics, Infectious Disease Unit, College of Medicine, King Saud University, King Saud University Medical City, Riyadh 11461, PO 2526 , Saudi Arabia (e-mail: ebukhari@ksu.edu.sa).
\end{abstract}

Abstract
Keywords
- Aeromonas hydrophila
- brain abscess
- preterm

Neonatal brain abscess is rare. The most common etiologic microorganisms that are responsible for this condition are Citrobacter diversus, Proteus mirabilis, Pseudomonas aeruginosa, Serratia marcescens, and other Enterobacteriaceae. Aeromonas hydrophila is a halophilic, gram-negative, facultative anaerobic bacillus. It has been reported to cause intestinal and extraintestinal infections and has rarely been reported to cause meningitis at neonatal age. A. hydrophila has not been reported previously to cause brain abscess in neonates. In this report, we describe a case of severe multiple brain abscesses and ventriculitis as complications in meningitis caused by A. hydrophila in a preterm newborn.

\section{Introduction}

The patient was a male newborn with a birth weight of $1.23 \mathrm{~kg}$ who was the first of twins. The mother was a 29-year-old female with a known case of sickle cell anemia. She had a history of pneumonia 3 weeks before delivery with twin pregnancy. The baby was delivered by vaginal delivery and was admitted to the neonatal intensive care unit. Blood was sent for culture and microbial/pathological analysis and then empirical antibiotic therapy was started (including intravenous ampicillin and gentamicin). After 7 days, the cultures were found negative, so the antibiotics were stopped. At the age of 31 days, the newborn had few episodes of desaturation and bradycardia, so septic workup was done. The first blood culture was sterile, while cerebrospinal fluid (CSF) was negative. As the baby was stable, no antibiotics were given. However, the baby became lethargic after 48 hours with bradycardia, but no bulging of the fontanelles was noticed, and there were no focal neurological signs. Sepsis was suspected, and samples of blood and CSF were sent for analysis and culture. The complete blood count showed white blood cell (WBC) count of $35,500 \mu \mathrm{L}\left(5.0-20.0 \times 10^{9} / \mathrm{L}\right)$ and C-reactive protein of $119 \mathrm{mg} / \mathrm{L}(0-10 \mathrm{mg} / \mathrm{L})$, and the blood culture was positive for gram-negative organism identified as Aeromonas hydrophila; the CSF showed the following results: WBC of $4,000\left(>15 / \mathrm{mm}^{3}\right)$, red blood cell (RBC) of 570 (cells $/ \mu \mathrm{L}$ ), $90 \%$ polymorph, low glucose of $14 \mathrm{mg} / \mathrm{dL}(50-80 \mathrm{mg} / 100 \mathrm{~mL})$, and CSF protein at $90 \mathrm{mg} / \mathrm{dL}(15-60 \mathrm{mg} / 100 \mathrm{~mL})$. No organisms were seen in Gram staining. The CSF culture grew gramnegative bacilli. The bacterium was biochemically identified as A. hydrophila, which was confirmed by Vitek. Antibiogram (a system used for fast, accurate microbial identification, and antibiotic susceptibility testing) revealed that the isolate was sensitive to cefotetan, imipenem, meropenem, amikacin and resistant to ampicillin and piperacillin-tazobactam; the antibiotics were changed to amikacin ( $18 \mathrm{mg} / \mathrm{kg} /$ dose q48 hour) and meropenem ( $40 \mathrm{mg} / \mathrm{kg} /$ dose $\mathrm{q} 8$ hour) at meningitic doses. After 72 hours on antibiotics, CSF analysis and culture were repeated, which revealed the following: RBC: $20 / \mu \mathrm{L}, \mathrm{WBC}$ : $3,300 / \mu \mathrm{L}$, polymorph nuclear leukocytes: $80 \%$, and lymphocytes: $20 \%$. Both Gram stain and culture were negative. Magnetic resonance imaging of the brain was done ( - Fig. 1A, B) and revealed the following: There were about 12 ring-enhanced lesions noted in bilateral cerebral hemispheres. All the lesions show homogeneous smooth enhancement of the wall with corresponding diffusion restriction and received

May 25, 2018

accepted after revision

July 30, 2018
DOI https://doi.org/

10.1055/s-0038-1669475. ISSN 2474-5871.
Copyright $\odot 2018$ Georg Thieme Verlag KG Stuttgart · New York
License terms

(요 (1) $\Theta \circledast$ 

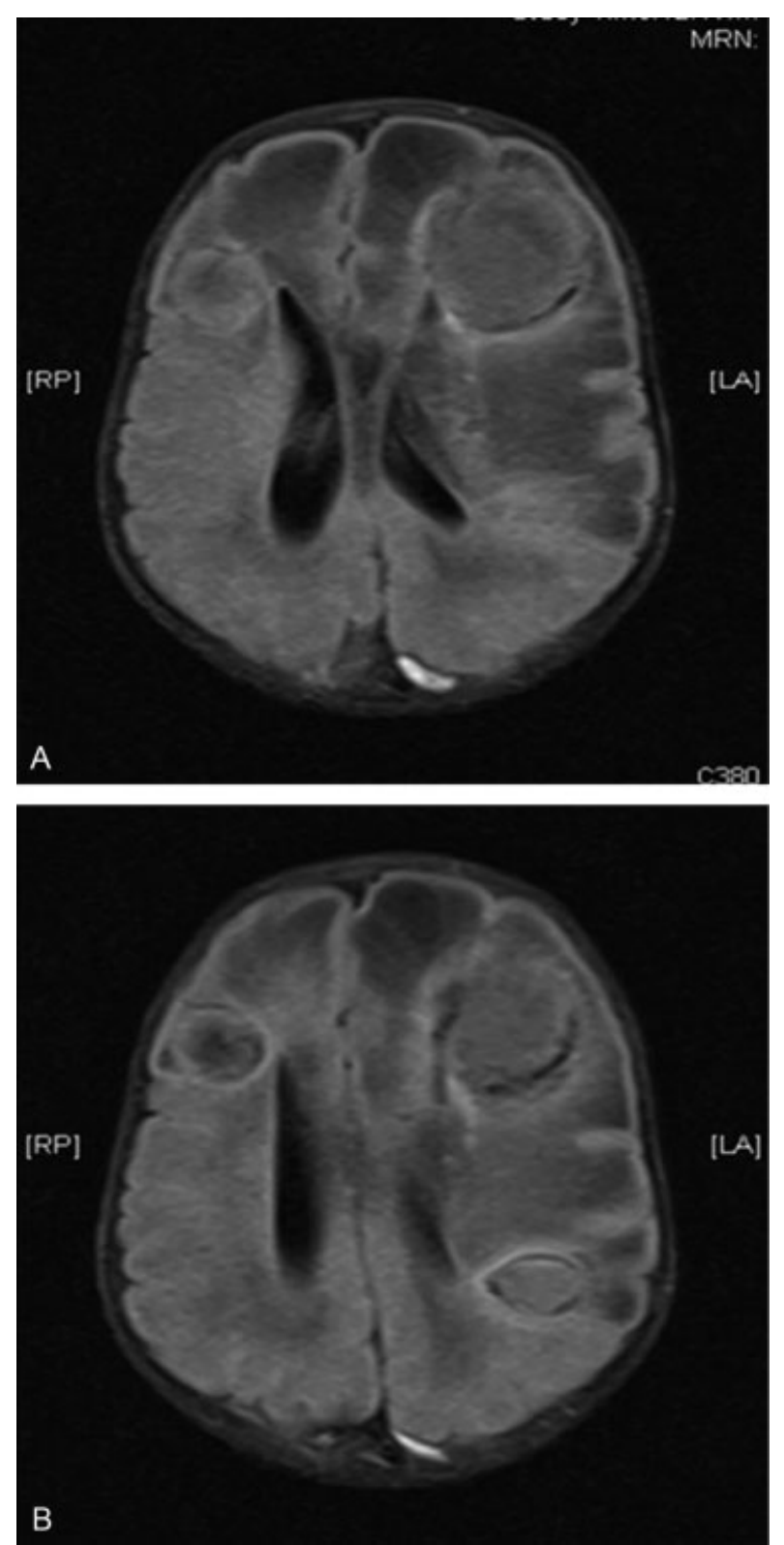

Fig. 1 (A, B) Magnetic resonance imaging of the brain revealed the following: There were multiple $(\sim 12)$ ring enhancing lesions noted in bilateral cerebral hemispheres, the largest one in left frontal lobe measures $4.5 \times 3.4 \times 2.2 \mathrm{~cm}$. The lesion in right parietal lobe shows clear direct rupture into the adjacent lateral ventricle. The findings are characteristic of multiple abscesses with ventriculitis and meningitis.

blooming on susceptibility-weighted imaging indicating hemorrhagic changes. Extensive surrounding edema is noted around the lesions. Edema extends to almost both cerebral hemispheres with relative sparing of the right posterior frontal and temporal lobes.

The lesion in right parietal lobe shows clear direct rupture into the adjacent lateral ventricle. Similar faint extension from left frontal lobe lesion is also seen. Diffuse enhancement of the ependyma of the ventricular system indicating ventriculitis is also seen. No diffusion restricted material is noted within the ventricles. Extensive leptomeningeal enhancement is noted, more obvious around brainstem and cerebellar folia.
Neurosurgeon was consulted; surgical evacuation of the largest frontal abscess was done. We attempted to identify the source of the infection. Several environmental samples were screened by culture, including apparatuses and water sources in the ventilator. However, A. hydrophila could not be recovered from these samples. During the same week, another baby in another room had positive culture for A. hydrophila from tracheal aspirate. The baby was treated with antibiotics for a total of 12 weeks.The baby developed hydrocephalus as a sequel of brain abscess; he is under follow-up by the neurosurgeon.

\section{Discussion}

Brain abscess is one of the most critical complications of bacterial meningitis in neonates. ${ }^{1}$ There are many bacterial pathogens reported to cause brain abscess.A. hydrophila has not been reported previously to cause brain abscess in neonates. ${ }^{2-4}$ Aeromonas species have been reported as a cause of extraintestinal infections such as skin and soft tissue infections, cholangitis, necrotizing fasciitis, and septicemia, especially in immunocompromised patients. ${ }^{5,6}$ Infections of the central nervous system secondary to $A$. hydrophila are relatively rare, and only a few cases of meningitis in infants and children have been reported. Some of the cases of meningitis were reported in association with sickle cell anemia, $\beta$-thalassemia, and hemoglobin., ${ }^{7,8}$

Kali et al reported clinical findings including episodes of apnea, bradycardia, hypotension, and seizures. ${ }^{2}$ Other clinical manifestations were absent, such as diarrhea, temperature instability, nuchal rigidity, bulging of the fontanelles, and focal signs. This is in the conformity with our findings. Our patient had a sepsis-like clinical picture with bradycardia and apnea but no bulging anterior fontanelle or meningeal signs.

The ideal management of $A$. hydrophila meningitis is unknown. A. hydrophila is resistant to commonly used drugs such as penicillin, ampicillin, and first-generation cephalosporin. However, it is susceptible to extended-spectrum cephalosporin, carbapenems, fluoroquinolones, aminoglycosides, and chloramphenicol. We treated our patient with meropenem and do not prefer to use third-generation cephalosporin to avoid the development of resistance. ${ }^{9}$ Similar to other reported cases of meningitis, we could not isolate the pathogen from environmental samples. ${ }^{2,9}$

\section{Funding}

This project was supported by the College of Medicine Research Centre, Deanship of Scientific Research, King Saud University, Riyadh, Saudi Arabia.

\section{References}

1 Sundaram V, Agrawal S, Chacham S, Mukhopadhyay K, Dutta S, Kumar P. Klebsiella pneumoniae brain abscess in neonates: a report of 2 cases. . J Child Neurol 2010;25(03):379-382

2 Kali A, Kalaivani R, Charles P, Seetha KS. Aeromonas hydrophila meningitis and fulminant sepsis in preterm newborn: A case report and review of literature. . Indian J Med Microbiol 2016;34 (04):544-547 
3 Traoré P, Coquery S, Zupan-Simunek V, Guibert M, Boileau P. Multiple brain abscesses complicating Enterobacter cloacae sepsis in a premature infant [Article in French]. Arch Pediatr 2010;17 (Suppl (Suppl 4):S184-S187

4 Heep A, Schaller C, Rittmann N, Himbert U, Marklein G, Bartmann P. Multiple brain abscesses in an extremely preterm infant: treatment surveillance with interleukin-6 in the CSF. Eur J Pediatr 2004; 163(01):44-45

5 Janda JM, Abbott SL. The genus Aeromonas: taxonomy, pathogenicity, and infection. . Clin Microbiol Rev 2010;23(01):35-73
6 Mukhopadhyay C, Chawla K, Sharma Y, Bairy I. Emerging extraintestinal infections with Aeromonas hydrophila in coastal region of southern Karnataka. . J Postgrad Med 2008;54(03):199-202

7 Seetha KS, Jose BT, Jasthi A. Meningitis due to Aeromonas hydrophila. . Indian J Med Microbiol 2004;22(03):191-192

8 Sirinavin S, Likitnukul S, Lolekha S. Aeromonas septicemia in infants and children. . Pediatr Infect Dis 1984;3(02):122-125

9 Mahabeer Y, Khumalo A, Kiratu E, Mlisana K. Posttraumatic brain abscess caused by Aeromonas hydrophila. . J Clin Microbiol 2014;52(05):1796-1797 